\title{
Von Nervenkitzel, Notfällen und Nobelpreisen
}

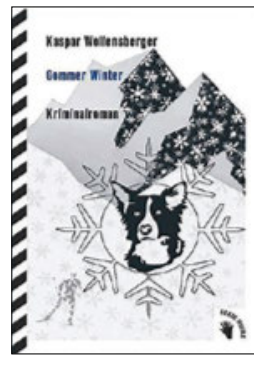

gnügt die Hände reiben. Wie Kauz sich entscheidet, bleibt offen. Vielleicht erfahren wir es im Frühjahr.

Dr. med. Erhard Taverna

Kaspar Wolfensberger

Gommer Winter

Kriminalroman

Zürich: bilgerverlag; 1. Auflage 2017

ISBN: 978-3037620694

477 Seiten. 36.00 CHF.

Nach den orkanartigen Stürmen und Schneefällen von Burglind und Christine im Januar 2018 waren viele Seitentäler im Wallis durch Schneemassen abgeschnitten. In einer ebenso klaustrophobischen Atmosphäre endet der neue Kriminalroman von Kaspar Wolfensberger. Kauz, der vom Gommer Sommer bekannte Ermittler von der Zürcher Kriminalpolizei, ist bis Ende Jahr freigestellt. Dann muss er sich entscheiden, zurück an den alten Posten oder eine Anstellung im Walliser Polizeikorps. Beim Showdown ist die Stromversorgung ausgefallen, der Akku vom Handy ist leer, Lawinen bedrohen die ganze Talschaft. Und in Münster läuft ein Serienmörder frei herum. Bis zur nervenkitzelnden Auflösung der Geschichte lernen die Leser das ausgedehnte Wegnetz und die vielen Loipen im Obergoms kennen. Hund Max folgt seinem Herrn auf ausgedehnten Waldläufen und beim Auffrischen der Langlauftechnik. Ein Mord geschieht und dann noch einer, und schliesslich ist es ein Jahre zurückliegender Todesfall, der die losen Enden verbindet. Kaspar Wolfensberger, der Psychiater und Schriftsteller aus Zürich, strickt einmal mehr kunstvoll mehrere Erzählstränge zu einem farbigen Teppich mit den ewig gleichen Mustern von Eifersucht, Hass und Rache. Das wird episch und detailreich ausgebreitet, ist aber dank der Beobachtungsgabe und Fabulierkunst des Autors von Anfang bis Ende spannend zu lesen. Auch wer den Vorläuferkrimi nicht kennt, hat keine Mühe, sich zurechtzufinden. Nicht nur Langlauffans, Wanderfreunde, Bergfreaks und krimilesende Ofenhocker dürften am Buch Gefallen finden. Auch der Gomser Tourismusdirektor wird sich ver-

Notfälle in der Hausarztpraxis, Von Allergie bis Zeckenbiss

Göttingen: Hogreve Verlag; 2., aktualisierte und ergänzte Auflage 2017.

ISBN: 978-3-456-85834-0

104 Seiten. 29.90 CHF.

Man möchte dieses praktische Handbuch geradezu als Pflichtlektüre allen empfehlen, die in ihrem Alltag mit medizinischen Notfällen konfrontiert werden, so zum Beispiel Eltern, Lehrpersonen jeglichen Berufes, natürlich unseren Medizinischen Praxisassistentinnen und nicht zuletzt uns Ärzt/innen. Auch mit dieser zweiten Auflage ist es dem Autor und langjährig erfahrenen Haus- und Lehrarzt Dr. med. Felix Schürch gelungen, verschiedene, potentiell lebensbedrohliche Situationen kurz und prägnant zu schildern. Es mag einem vielleicht seltsam vorkommen, einen Zeckenbiss als «Notfall» zu bezeichnen. Die Betroffenen jedoch können das durchaus als solchen empfinden und drängen auf einen raschen Termin, hat man doch schon so viel darüber gehört, bis hin zur schweren Lähmung kann das gehen, wenn man nicht das Richtige rechtzeitig getan hat! So sind dann - vor allem vorne beim Empfang und besonders am Telefon beim Terminieren - unsere Medizinischen Praxisassistentinnen sehr gefordert. Sie müssen lernen, diese Situationen richtig einzuschätzen, allenfalls $\mathrm{zu}$ beruhigen oder eben zeitnah irgendwie noch einen Termin zu vergeben. Genau dies so treffend wie möglich einzuschätzen ist unter anderem die grosse Kunst, Erfahrung und Verantwortung unserer Sprechstunden-Assistentinnen. Dabei hilft dieses Handbuch. Die alpha- betische Inhaltsangabe zu Beginn ermöglicht das Auffinden der Themenbereiche. Ein Index zum Ende würde das rasche Auffinden von Stichworten erleichtern. Im Volltext nach der Antwort auf eine dringende Frage zu suchen, kann so gerade in einem "richtigen" Notfall doch recht stressig werden.

Dr. med. Frank Wyler-Brem

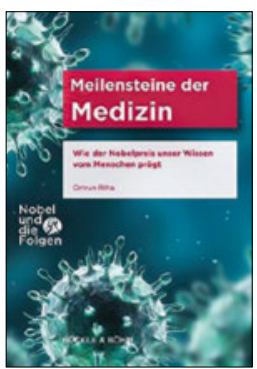

\section{Ortrun Riha}

Meilensteine der Medizin. Wie der Nobelpreis unser Wissen vom Menschen prägt

Regensburg: Bückle \& Böhm; 2016.

ISBN: 978-3-941530-11-9

237 Seiten. 88 Abbildungen. CHF 35.90.

Der Nobelpreis gilt als die prestigeträchtigste Auszeichnung für einen Forscher in der Medizin. Deshalb ist es nicht verwunderlich, dass es eine beinahe unüberschaubare Fülle an Literatur rund um den Preis gibt, darunter auch zahlreiche historische Publikationen.

Die Leipziger Medizinhistorikerin Ortrun Riha kontextualisiert in fünf ausführlichen Kapiteln wesentliche Fächerschwerpunkte der bisherigen Nobelpreislaureaten für Physiologie oder Medizin, darunter die der Schweizer Preisträger Theodor Kocher (Nobelpreis 1909) und Rudolf W. Hess (Nobelpreis 1949). Lobenswert sind auch die Essays, die über die faktischen Nobelpreisträger hinausgehen. Dabei werden Themen wie "Preiswürdige Leistungen ohne Preis", "Obsolete Ergebnisse und problematische Forschungen» oder «Nobelpreisträger als Philosophen» erörtert. Das bilderreiche Buch liefert einen spannenden Streifzug durch die Nobelpreisgeschichte seit der ersten Preisverleihung 1901 und ist einem breiten Ärztepublikum zu empfehlen.

Dr. Nils Hansson 